\title{
Equivalent local potentials to multiple scattering calculations of nucleon-nucleus scattering
}

\author{
R. Crespo \\ Departamento de Física, Instituto Superior Técnico, Universidade Técnica de Lisboa, \\ Avenida Rovisco Pais, P-1096 Lisboa, Portugal \\ and Centro de Física Nuclear, Universidade de Lisboa, Avenida Prof. Gama Pinto 2, P-1699 Lisboa, Portugal \\ R. C. Johnson and J. A. Tostevin \\ Department of Physics, University of Surrey, Guildford, Surrey GU2 5XH, United Kingdom \\ R. S. Mackintosh and S. G. Cooper \\ Physics Department, The Open University, Milton Keynes, MK7 6AA, United Kingdom
}

(Received 23 February 1993)

\begin{abstract}
Local phase equivalent interactions to the nonlocal Kerman, McManus, and Thaler multiple scattering nucleon-nucleus optical potentials are determined. Both first- and second-order contributions in the free nucleon-nucleon transition amplitude are included. The second order terms modify the derived potentials in a nontrivial way and reduce the strengths of the real and imaginary central potentials in the nuclear interior. The nonlocality of the first- and second-order multiple scattering potentials is quantified by the evaluation of an associated Perey factor. Calculations give a mean free path of $\lambda=3-4 \mathrm{fm}$ for $100-200 \mathrm{MeV}$ incident nucleons on ${ }^{16} \mathrm{O}$.
\end{abstract}

PACS number(s): 24.10.Ht, 24.70.+s, 25.40.Cm

\section{INTRODUCTION}

The interpretation of a large body of experimental data involving hadronic initial or final states, such as $\left(p, p^{\prime}\right)$ and $\left(e, e^{\prime} p\right)$ reactions, has as a fundamental input a knowledge of nucleon propagation in the nuclear medium. Such propagation is often characterized in terms of the nucleon mean free path $\lambda$, a measure of the average distance between nucleon-nucleon $(N N)$ collisions. This nucleon propagation or distortion is usually included in quantitative reaction calculations through the use of local phenomenological nucleon-target potentials, single scattering impulse approximation potentials, or approximate $G$-matrix based interactions. In this work we will investigate the extent to which the second-order contributions to the Kerman, McManus, and Thaler (KMT) [1] optical potential modify the single scattering approximation expectations of the nucleon-target interaction and the resulting distorted wave functions. We quantify these modifications through the calculated nucleon mean free paths $\lambda$ and an associated Perey factor, respectively.

The first-order term of the nonrelativistic multiple scattering expansion of the optical potential in terms of the free $N N$ transition amplitude, such as developed by Kerman, McManus, and Thaler [1], has recently been the subject of several very detailed analyses. These works paid particular attention to the nonlocalities inherent of the interaction [2-5] and as a result the numerical calculations were most readily carried out in momentum space. A comprehensive review of both the relativistic and nonrelativistic descriptions of the nucleon-nucleus system can be found in [6]. The nonrelativistic KMT potential to first order in the $N N$ transition amplitude will be referred to as the single scattering approximation (SSA). In local " $t \rho$ " approximations to the SSA, the shape of the optical potential is determined primarily by that of the target density for both the real and the imaginary central terms of the interaction.

Recently, new calculations of the KMT nucleonnucleus optical potential have been carried out [7] which include terms in the multiple scattering series to second order in the free $N N$ transition amplitude. These will be referred to as the double scattering approximation (DSA) to the optical potential. The inclusion of the secondorder terms modifies the optical potential obtained using the free NN transition amplitude appearing in the SSA by accounting for Pauli blocking medium effects due to the identity of the struck and core nucleons [7]. In the DSA calculations of Ref. [7], for proton scattering from ${ }^{16} \mathrm{O}$ at $100-200 \mathrm{MeV}$ incident energy, the nonlocalities of the optical potential arising from both the NN transition amplitude and from the propagation of the nucleon in intermediate states between scattering events are included. These calculations were also performed in momentum space. In these calculations, in the NN amplitude, the potential energy felt by the struck nucleon is neglected. A recent estimate of these binding corrections, expressed as a momentum-transfer-dependent energy shift of the energy of the NN transition amplitude, has been presented elsewhere [8].

Although the DSA calculations provide improvement in the description of the empirical reaction cross section over the SSA [7], the theoretical DSA optical potentials, like those of the SSA, remain far from satisfactory in their description of the available elastic scattering data. The quality of the agreement with data at $200 \mathrm{MeV}$ can be 
assessed by inspection of the figures of Ref. [3] and Fig. 7 of Ref. [7]. Further theoretical refinements are known to be required. In particular, a consistent treatment of all aspects of the nuclear medium [8] on the interacting $N N$ pair, in the SSA, will be required before definitive quantitative conclusions can be drawn. Our purpose here is to obtain estimates of the magnitudes of the corrections to the nucleon propagation to be expected to reaction calculations either which (i) make use of SSA theoretical optical potentials, by comparison of the DSA and SSA mean free paths and Perey factors, or which (ii) make use of local optical potentials, through a study of the DSA Perey factor.

To gain insight into the momentum space multiple scattering calculations, specifically of the magnitudes and shapes of the refractive and reactive terms of the optical potential, and to clarify the changes induced by the second order KMT terms, it is instructive to evaluate and study the form of local equivalent configuration space potentials. These phase equivalent local interactions will be chosen to reproduce with high accuracy the elastic partial wave $S$-matrix elements obtained from the full microscopic (momentum space) calculations. With regard to the practice of using local optical potentials in reaction calculations, we are interested in the extent to which the elastic $S$-matrix elements of the KMT potential are capable of being reproduced in detail by a smooth and orbital angular momentum independent local interaction. This being the case the elastic wave functions are readily generated for use in reaction calculations. However, the question of the modifications to the wave functions within the nuclear volume resulting from the nonlocalities of the optical potential remains. We quantify these modifications by calculation of an associated Perey factor

In order to avoid the complications arising from the discussion of the treatment of the Coulomb interaction in the momentum space calculations, in the present work we consider only the strong interaction component of the nucleon-nucleus interaction. Recently Cooper [9], using the Dirac phenomenology approach, obtained phenomenological nucleon-nucleus optical potentials by fitting a wide range of experimental data in the intermediate energy region; much extending to large scattering angles. The real part of the potentials so obtained had a Woods-Saxon form, while the imaginary part required to be surfaced peaked in order to reproduce the experimental data. As was noted above, in the KMT approach, local " $t \rho$ " approximations produce potentials which follow the target density and cannot derive such a surface behavior. In the present work we are able to investigate the qualitative behavior of the modifications to the first-order optical potential induced by the DSA Pauli blocking effects.

\section{NONLOCAL POTENTIAL CALCULATIONS}

In the following, the derivation of the local equivalent potentials and of the Perey factors require as inputs the elastic partial wave $S$-matrix elements and the configuration space radial wave functions generated by the non- local KMT optical potentials, respectively. For the scattering of a spin $s=\frac{1}{2}$ particle, of incident spin projection $\sigma$, from a spinless target of mass $A$ we define the configuration space partial wave decomposition of the scattering wave function in the absence of the Coulomb interaction by

$$
\begin{array}{rl}
\psi_{\sigma}^{(+)}\left(\mathbf{k}_{0}, \mathbf{r}\right)=\frac{1}{k_{0} r} \sum_{L \lambda J M} & 4 \pi i^{L} u_{L J}(r) Y_{L \lambda}^{*}\left(\hat{\mathbf{k}}_{0}\right) \\
\times(L \lambda s \sigma \mid J M) \mathcal{Y}_{(L \boldsymbol{s}) J}^{M}(\hat{\mathbf{r}}),
\end{array}
$$

where $k_{0}$ is the incident wavenumber, $\mathcal{Y}_{(L s) J}^{M}$ is the spinangle function, and $Y_{L \lambda}$ is the spherical harmonic. The radial wave functions $u_{L J}(r)(J=L \pm 1 / 2)$ have the asymptotic form

$$
u_{L J}(r) \rightarrow \hat{\mathbf{j}}_{L}\left(k_{0} r\right)+T_{L J}(N) \hat{\mathbf{h}}_{L}^{+}\left(k_{0} r\right),
$$

where the $T_{L J}(N)=\exp \left(i \delta_{L J}\right) \sin \delta_{L J}$ are the partial wave transition amplitudes due to the nuclear interaction and $\hat{\mathbf{h}}_{L}^{+}=\hat{\mathbf{n}}_{L}+i \hat{\mathbf{j}}_{L}$ with $\hat{\mathbf{j}}_{L}$ and $\hat{\mathbf{n}}_{L}$ the Riccati-Bessel and Neumann functions, $\hat{\mathbf{j}}_{L}(x)=x j_{L}(x)$, etc.

In the KMT multiple scattering calculations, however, we solve the Lippmann-Schwinger equation, in momentum space, for the auxiliary transition amplitude [1] $T_{L J}^{\prime}$, where

$$
T_{L J}^{\prime}(N)=\left(\frac{A-1}{A}\right) T_{L J}(N) .
$$

In generating the local phase equivalent potentials to the multiple scattering potentials the partial wave $S$-matrix elements to be reproduced are therefore

$$
S_{L J}=1+2 i\left(\frac{A}{A-1}\right) T_{L J}^{\prime}(N)
$$

The configuration space radial wave functions of the nonlocal (NL) KMT interaction $u_{L J}^{\mathrm{NL}}(r)$ are calculated from the half-shell nucleon-nucleus transition amplitudes $T_{L J}^{\prime}\left(k, k_{0}\right)$ using the method of Eisenstein, Tabakin, and Landau $[10,11]$ in the absence of the Coulomb interaction. We have found that this method reproduces accurately the wave function obtained using conventional configuration space methods in a number of test cases at the energies of interest.

\section{LOCAL EQUIVALENT POTENTIALS}

To obtain the phase equivalent local potentials we exploit the iterative perturbative (IP) $S$-matrix to potential inversion procedure pioneered by Mackintosh and coworkers [12]. The method, subsequently developed by Cooper and Mackintosh [13], is now available as code IMAGO [14] for practical calculations of a spin- $\frac{1}{2}$ particle scattering from spinless target nuclei. We quantify the accuracy of each inversion calculation by means of the distance measure $\delta$ defined by

$$
\delta^{2}=\sum_{L, J}\left|S_{L J}^{\mathrm{tar}}-S_{L J}^{\mathrm{inv}}\right|^{2}
$$


where the $S_{L J}^{\text {tar }}$ are the "target" $S$-matrix elements, in our case the KMT nonlocal potential results, and the $S_{L J}^{\text {inv }}$ are those of the local equivalent potential obtained by inversion. All of the local potentials obtained, the results of which are quoted in this work, satisfy $\delta<0.002$. The radial behavior of the resulting local potentials is also found to be completely insensitive to details of the basis states chosen for the representation of the interactions within the IP procedure (e.g., spline, Gaussian, or Bessel function expansions). We present the principal features of these local inverted potentials, in particular we analyze the effects of the second-order KMT optical potential contributions on the shapes and magnitudes of the local potential form factors.

Figure 1 shows the local potential results for nucleon${ }^{16} \mathrm{O}$ scattering at $100 \mathrm{MeV}$. The solid (dashed) curves represent the local equivalent potentials calculated from the DSA (SSA) approximations to the optical potential. Specifically, the SSA calculations use the optimal factorization form of the optical potential given by Eqs. (47-52) of [7] and which assume the $N N$ transition amplitude is calculated at a fixed energy. The DSA potentials correspond to the fully nonlocal calculations of the secondorder KMT terms and include nonlocalities arising from both the NN transition amplitude and the propagation of the nucleon in intermediate states between scattering events. The potentials are defined by Eqs. (58-78) of Ref. [7]. Figure 2 shows the corresponding potentials at 200 $\mathrm{MeV}$ and demonstrates the already significant reduction in the importance of the Pauli blocking effects at 200 $\mathrm{MeV}$. In Fig. 3 we present the differences

$$
\Delta U(r)=U^{\mathrm{DSA}}(r)-U^{\mathrm{SSA}}(r),
$$

between the second- and first-order local potentials at

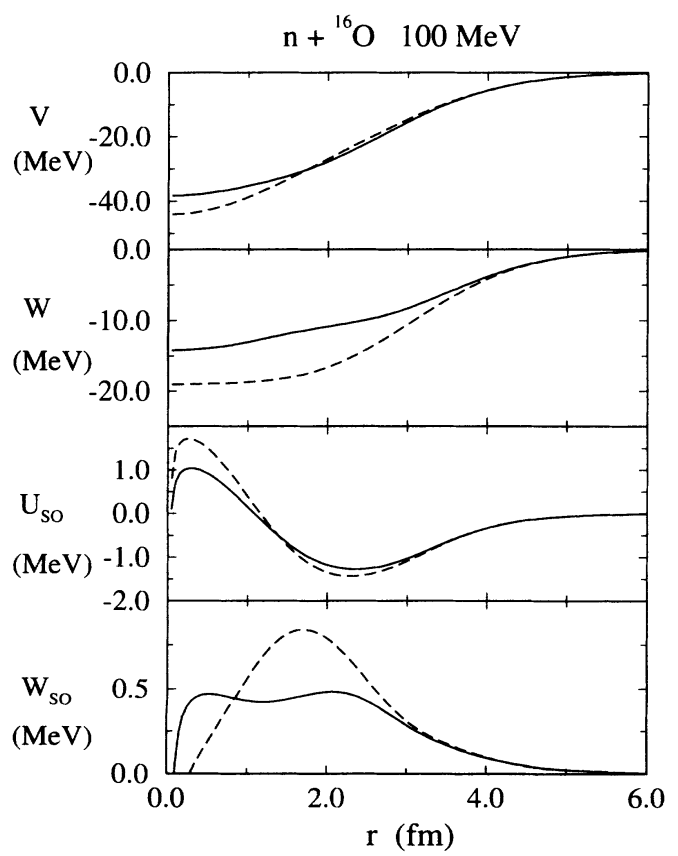

FIG. 1. Local equivalent potentials for nucleon- ${ }^{16} \mathrm{O}$ scattering at $100 \mathrm{MeV}$ predicted from the second-order (solid) and first-order (dashed) KMT optical potentials.

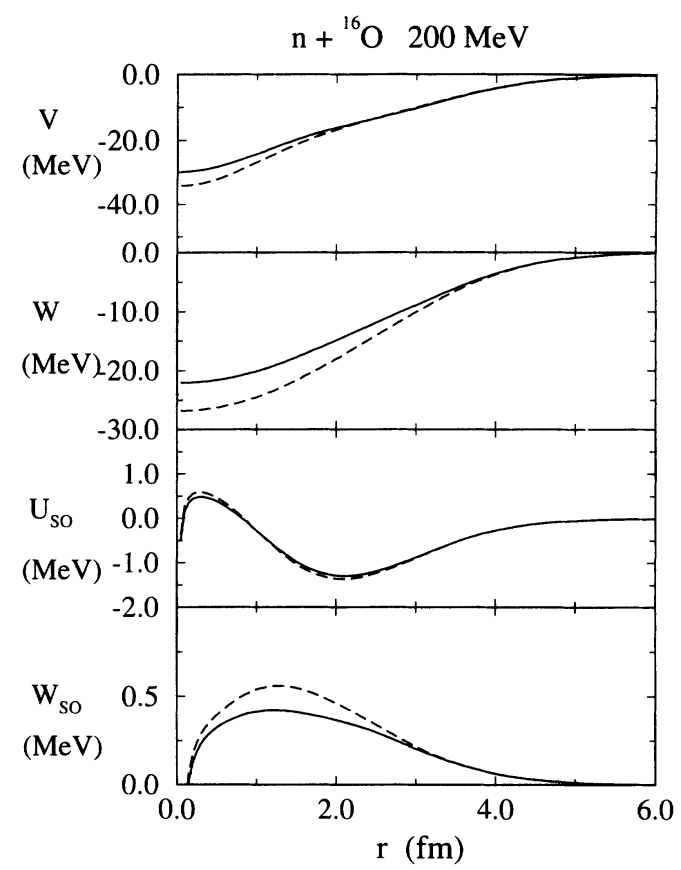

FIG. 2. Local equivalent potentials for nucleon $-{ }^{16} \mathrm{O}$ scattering at $200 \mathrm{MeV}$ predicted from the second-order (solid) and first-order (dashed) KMT optical potentials.

$100 \mathrm{MeV}$ (dashed), $135 \mathrm{MeV}$ (dot-dashed), and $200 \mathrm{MeV}$ (solid), and which are induced by the Pauli blocking terms. As shown in the figures, the effects of the secondorder terms are very significant in the nuclear interior particularly at the lower energies. This was to be anticipated since local approximations to the DSA terms have

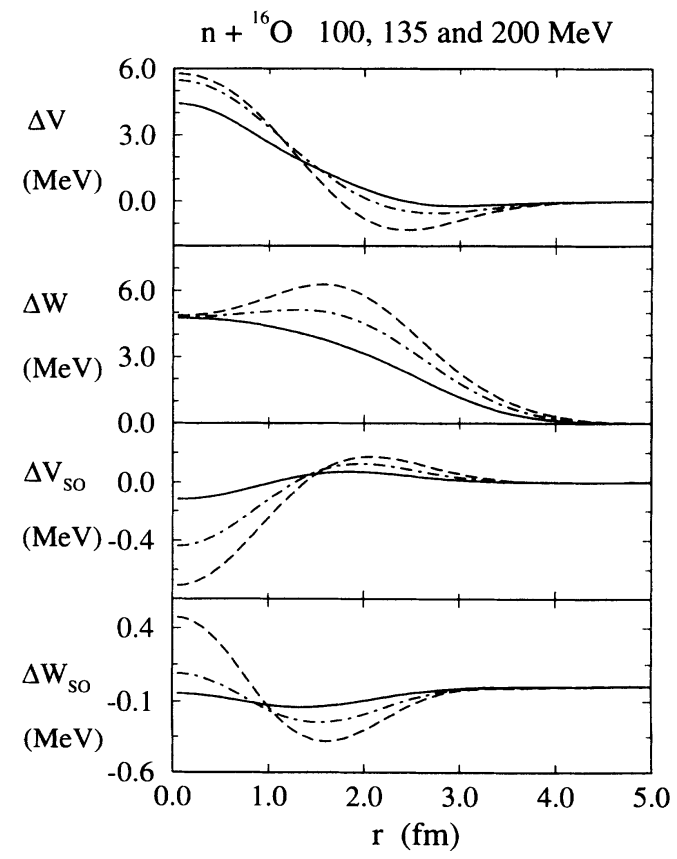

FIG. 3. Calculated differences between the local equivalent potentials of the second- and first-order KMT potentials for nucleon- ${ }^{16} \mathrm{O}$ scattering at 100 (dashed), 135 (dot-dashed), and $200 \mathrm{MeV}$ (solid). 
TABLE I. Calculated local potential volume integrals and rms radii at $100 \mathrm{MeV}$.

\begin{tabular}{cccc}
\hline \hline Order of the potential & Potential term & $\begin{array}{c}\left\langle r^{2}\right\rangle^{1 / 2} \\
(\mathrm{fm})\end{array}$ & $\begin{array}{c}J \\
\left(\mathrm{MeV} \mathrm{fm}^{3}\right)\end{array}$ \\
\hline \multirow{3}{*}{$\mathrm{SSA}$} & Real central & 3.26 & -328.8 \\
\cline { 2 - 4 } & Imaginary central & 3.32 & -224.2 \\
\hline \multirow{3}{*}{ DSA } & Real spin orbit & 3.32 & -17.7 \\
\cline { 2 - 4 } & Imaginary spin orbit & 3.06 & 7.1 \\
& Real central & 3.26 & -336.0 \\
\hline & Imaginary central & 3.47 & -180.6 \\
\cline { 2 - 4 } & Real spin orbit & 3.34 & -16.9 \\
\cline { 2 - 4 } & Imaginary spin orbit & 3.24 & 5.8 \\
\hline
\end{tabular}

a shape determined by the square of the target matter density. Since elastic scattering is overwhelmingly determined by the potentials in the nuclear surface, or the surface partial waves, the DSA terms are not expected to modify significantly the elastic observables [7]. The second-order calculations reduce the depth of both the real and imaginary parts of the central potential at the nuclear center. The Pauli blocking effects introduce comparatively smaller changes at the surface of the potential. We note that at the lowest energy the real part of the central potential becomes more diffuse and the DSA corrections to the imaginary part show a tendency to become surface peaked.

In Tables I and II we present the volume integrals and rms radii for the real and imaginary parts of the central and spin-orbit components of the local potentials at 100 and $200 \mathrm{MeV}$, respectively. As follows from the tables, the Pauli blocking medium effects in fact increase the real central volume integral very slightly at low energies since the $r^{2}$ weight in the volume integral probes the surface where the second-order terms lead to a slightly stronger interaction. The values obtained are considerably larger than the values predicted by nuclear matter calculations which are of magnitude $150-250 \mathrm{MeV} \mathrm{fm}^{3}$ and $100-150$ $\mathrm{MeV} \mathrm{fm}^{3}$ for the real and imaginary parts, respectively, in the energy range under consideration [15].

\section{PEREY FACTOR}

By comparing the actual configuration space wave functions $\psi^{\mathrm{NL}}$, generated from the momentum space cal- culations, and the wave functions $\psi^{\mathrm{LE}}$ obtained from the corresponding local equivalent potentials, information about the nonlocality of the optical potential can be obtained. Perey [16] demonstrated that for the phenomenological non-locality of Perey and Buck [17], the wave function $\psi^{\mathrm{NL}}$ is reduced in the nuclear interior compared with that associated with the equivalent local potential. The ratio of these wave functions is referred to as the Perey factor and such factors are a standard feature for the estimation of nonlocality corrections to distorted wave Born approximation calculations of transfer and inelastic processes. It is of interest therefore to investigate the way in which the wave functions of the full momentum space calculations, which embody the nonlocal effects considered herein exactly, behave within the nucleus when compared with the wave functions associated with the local phase equivalent potentials.

We use a generalized Perey factor $\mathcal{P}_{\sigma}(\mathbf{r})[18]$ (see also [19]), defined as the ratio

$$
\mathcal{P}_{\sigma}(\mathbf{r})=\frac{\left|\psi_{\sigma}^{\mathrm{NL}}\left(\mathbf{k}_{0}, \mathbf{r}\right)\right|}{\left|\psi_{\sigma}^{\mathrm{LE}}\left(\mathbf{k}_{0}, \mathbf{r}\right)\right|}
$$

of the moduli of the nonlocal and the local equivalent wave functions. These wave functions are complete partial wave sums so the $\mathcal{P}_{\sigma}$ contain angular as well as radial information concerning the Perey effect. We present the calculated $\mathcal{P}_{\boldsymbol{\sigma}}$ by means of contour plots.

In Figs. 4-7 the Perey factors $\mathcal{P}_{\sigma}(\mathbf{r})$ are shown for $\mathbf{r}$ values in the nucleon-nucleus scattering plane, defined by the directions of the incident, $\mathbf{k}_{0}$, and scattered, $\mathbf{k}_{f}, \mathrm{nu}$ cleon momenta. The incident beam direction $\mathbf{k}_{0}$ is from

TABLE II. Calculated local potential volume integrals and rms radii at $200 \mathrm{MeV}$.

\begin{tabular}{cccc}
\hline \hline Order of the potential & Potential term & $\begin{array}{c}\left\langle r^{2}\right\rangle^{1 / 2} \\
(\mathrm{fm})\end{array}$ & $\begin{array}{c}J \\
\left(\mathrm{MeV} \mathrm{fm}^{3}\right)\end{array}$ \\
\hline \multirow{3}{*}{$\mathrm{SSA}$} & Real central & 3.36 & -230.9 \\
\cline { 2 - 4 } & Imaginary central & 3.18 & -219.7 \\
\cline { 2 - 4 } & Real spin orbit & 3.19 & -15.6 \\
\cline { 2 - 4 } & Imaginary spin orbit & 2.98 & 4.6 \\
& & 3.38 & -229.1 \\
\hline \multirow{3}{*}{ DSA } & Real central & 3.25 & -195.7 \\
\cline { 2 - 4 } & Imaginary central & 3.20 & -15.3 \\
\cline { 2 - 4 } & Real spin orbit & 3.07 & 4.1 \\
\cline { 2 - 4 } & Imaginary spin orbit & & \\
\hline
\end{tabular}




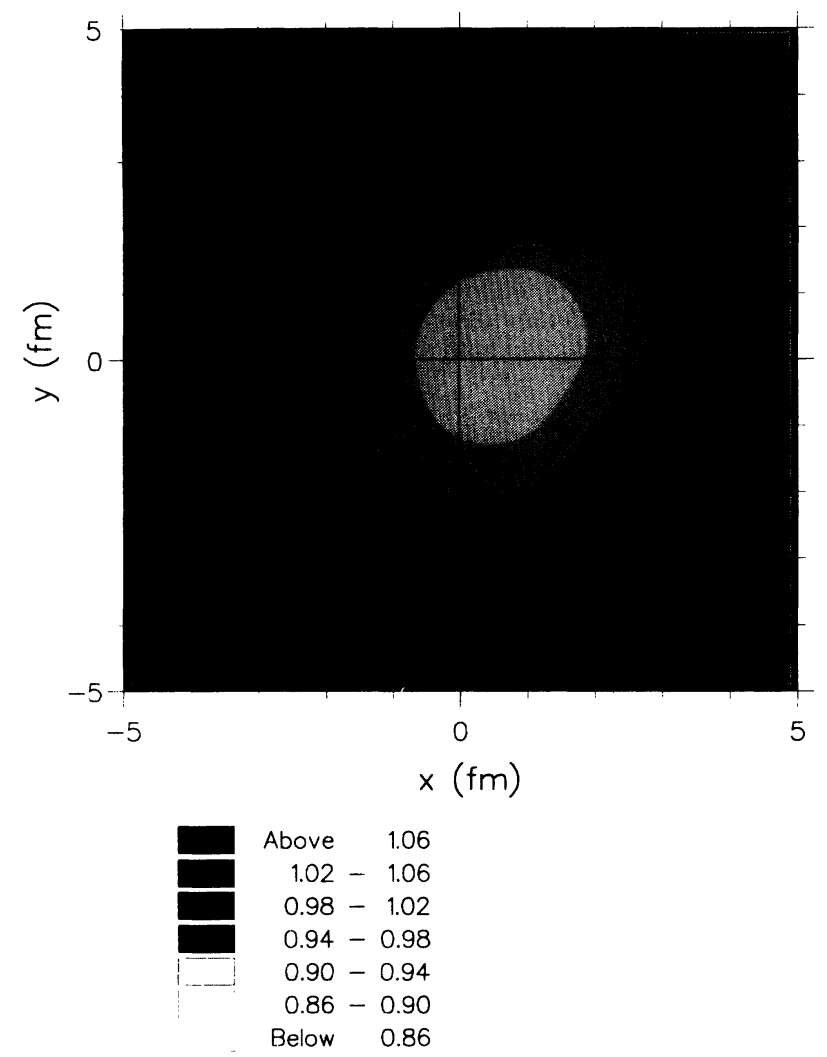

FIG. 4. Perey factor calculated from the first-order (SSA) optical potential at $100 \mathrm{MeV}$.

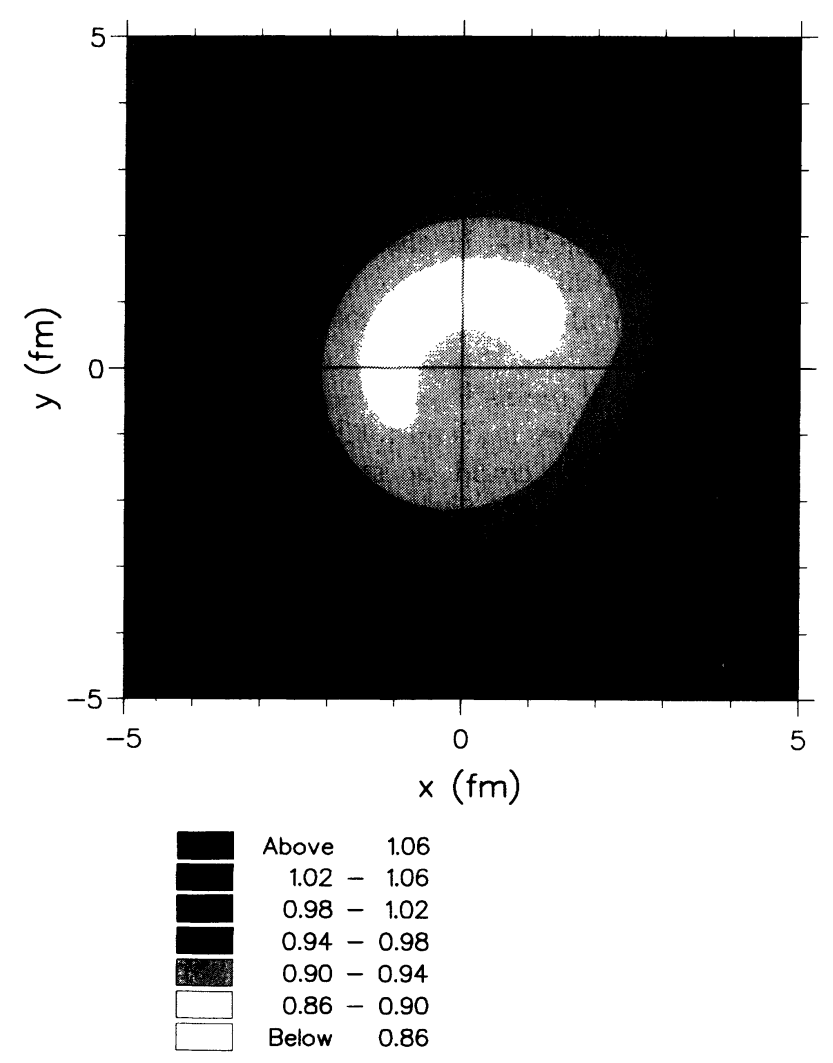

FIG. 5. Perey factor calculated from the second-order (DSA) optical potential at $100 \mathrm{MeV}$.

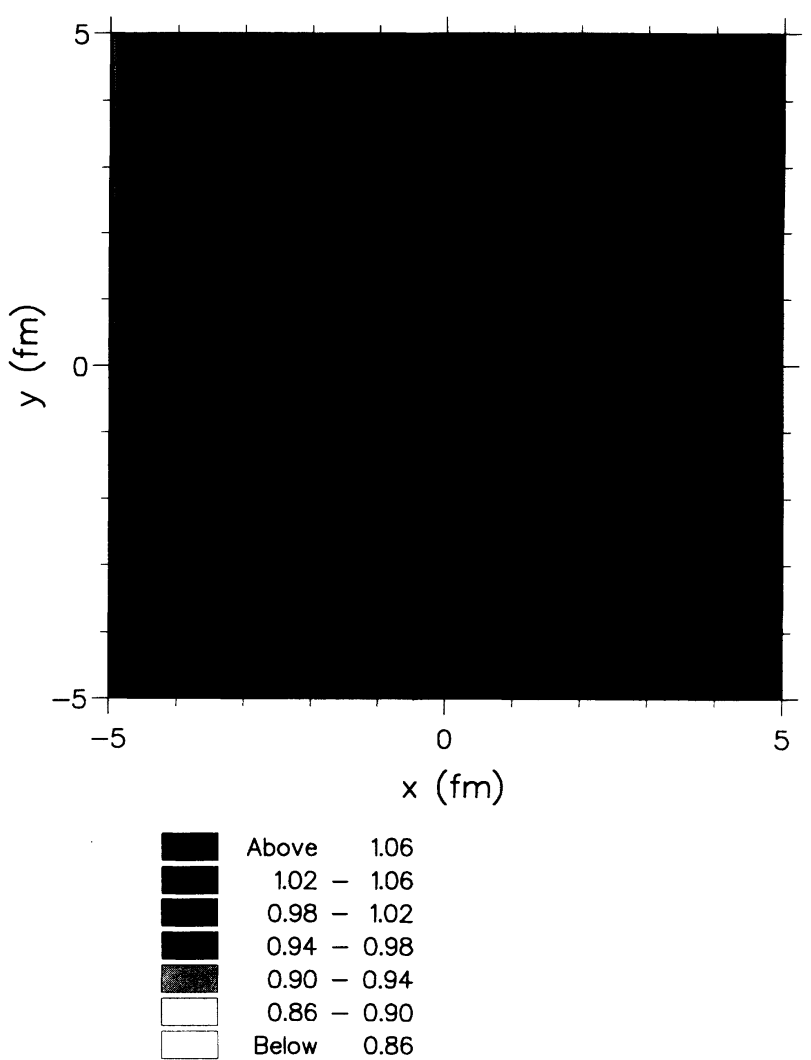

FIG. 6. Perey factor calculated from the first-order (SSA) optical potential at $200 \mathrm{MeV}$.

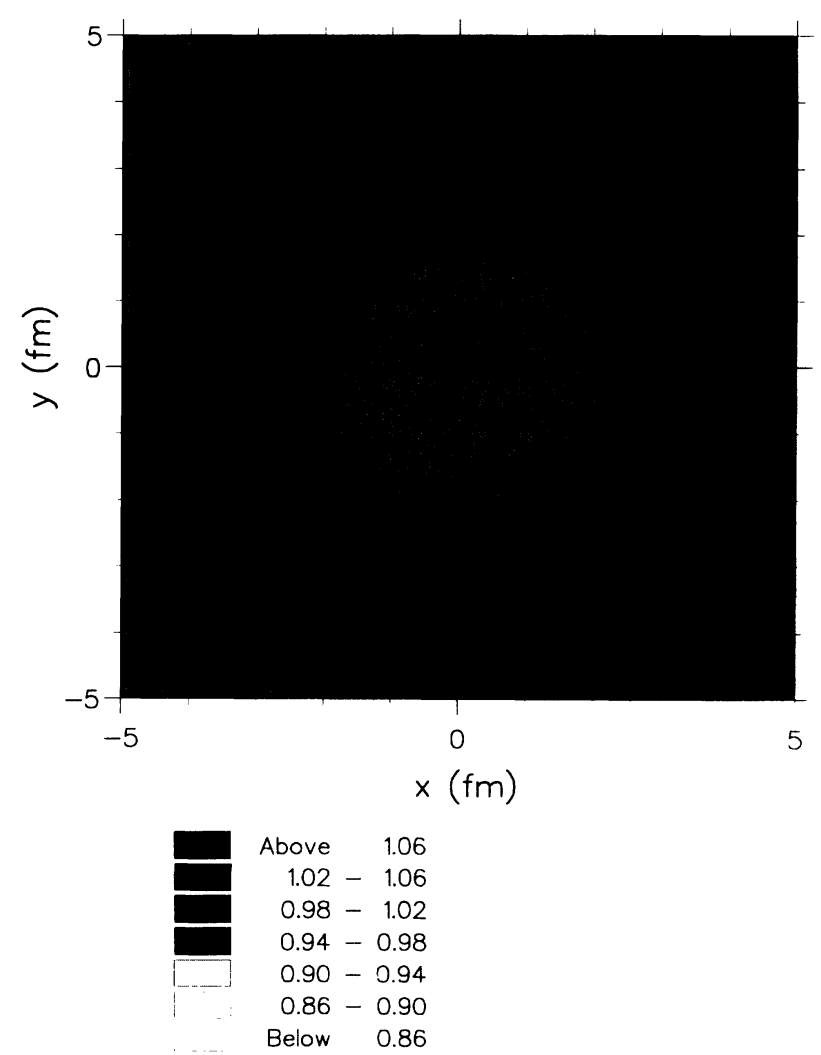

FIG. 7. Perey factor calculated from the second-order (DSA) optical potential at $200 \mathrm{MeV}$. 
left to right in the figures and parallel to the horizontal axis, which we may label the $x$ axis. The vertical axis on these figures, labeled the $y$ axis, thus represents the nucleon impact parameter. The target center is located at $(x, y)=(0,0)$. If $x$ and $y$ axes are so defined the $z$ axis of a right-handed coordinate system is now normal to, and directed out of the page, and is along the positive normal $\mathbf{k}_{0} \times \mathbf{k}_{f}$ for particles scattered to one side $(+y)$ of the beam. In Figs. 4-7 the calculations are for nucleons incident with spin projection $+\frac{1}{2}$ with respect to this normal to the scattering plane. The $\mathcal{P}_{\sigma}(\mathbf{r})$ presented can be constructed by setting the polar angles $\hat{\mathbf{k}}_{0}=(\pi / 2,0)$ and $\hat{\mathbf{r}}=(\pi / 2, \phi), \tan \phi=y / x$, and evaluating the partial wave sums in Eq. (1) at each point $\mathbf{r} \equiv(x, y, 0)$ in the scattering plane.

In Fig. 4 we show the Perey factor from the SSA optical potential at $100 \mathrm{MeV}$. Figure 5 shows the corresponding Perey factor from the DSA optical potential. In both SSA and DSA cases, the Perey factor becomes unity for distances greater than $4 \mathrm{fm}$ and reflects the phase equivalence of the wave functions in the asymptotic region. Particularly clear from Fig. 5 is that the $\mathcal{P}_{\sigma}$ can depart strongly from a circular form; expected for instance of a Perey-Buck type nonlocality. The lack of symmetry with respect to negative and positive values of the impact parameter reflects the inherent spin dependence in the Perey factor.

The construction of the Perey factor requires the radial wave functions $u_{L J}^{\mathrm{NL}}(r)$ of the momentum space calculations, as was described in Sec. II. The wave functions $u_{L J}^{\mathrm{LE}}(r)$ are provided directly by the configuration space inversion code IMAGO [14].

Although, by construction, the wave functions are identical in the asymptotic region, the local equivalent potential will not generate the same wave function at smaller radii. The departure of the Perey factor from unity then provides a measure of the modifications of the wave function in the nuclear interior and of the relative importance of the nonlocalities of the KMT optical potential. It should be noted that, as defined here, the Perey factor is a weighted contribution (essentially $2 L+1$ ) from all partial waves. This average measure of the nonlocality thus receives increased weight from the higher partial waves. Figure 4 shows that the nonlocalities associated with the SSA optical potential are relatively small with $\mathcal{P}_{\sigma}(r=0) \approx 0.86-0.90$. A somewhat different analysis of the Perey factor, in the case of the SSA, was also carried out in [3] with qualitatively similar results. As is evident from Fig. 5 the DSA calculations exhibit a significantly different effect. The Perey factor $\mathcal{P}_{\sigma}(\mathbf{r})<0.86$ at $100 \mathrm{MeV}$ over a range of $\mathbf{r}$ values and departs strongly from a circular form. The departure of the Perey factors from unity decreases rapidly as the incident beam energy increases as is demonstrated by the first- and second-order calculations at $200 \mathrm{MeV}$ shown in Figs. 6 and 7 , respectively.

The Perey factors found here are comparable in magnitude to the standard Perey factors often used at much lower energies. They therefore appear to represent a higher degree of nonlocality than is ordinarily expected in the energy range of this paper. In addition, the Perey

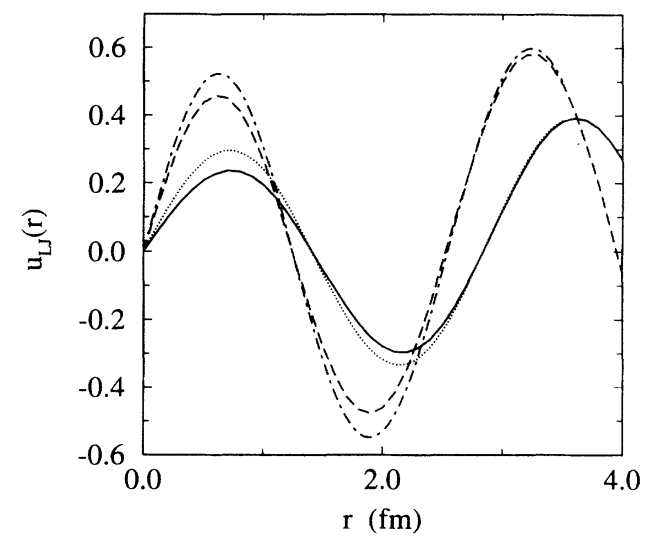

FIG. 8. Comparison of the calculated real (solid) and imaginary (dashed) parts of the radial wave functions from the second-order KMT potential and the real (dotted) and imaginary (dot-dashed) parts from the local equivalent potential in the $L=0, J=1 / 2$ partial wave at $100 \mathrm{MeV}$ incident nucleon energy.

factor associated with the medium effects of the secondorder KMT term cannot be represented by the radial correction factor usually incorporated in DWBA reaction calculations. We observe nontrivial modifications to both the angular and spin dependence as well as the radial dependence of the wave function.

The Perey factor defined by Eq. (7) is the averaged effect from all contributing partial waves, with increased weight from the higher partial waves. Since the nonlocal effects are known to be more significant in the lower partial waves [7], in processes sensitive to the wave function in the nuclear volume these nonlocalities are expected to exhibit stronger effects than are suggested by these average Perey factors. In Fig. 8 we illustrate the wave function modifications, $20 \%$ in the real part, in the $L=0$ partial wave at $100 \mathrm{MeV}$ by comparison of the nonlocal (DSA) and equivalent local potential configuration space wave functions.

The strength of inelastic and reaction processes which are sensitive to the nucleon-nucleus wave function in lower partial waves could be strongly affected by this modified propagation of the nucleon in the nuclear medium.

\section{MEAN FREE PATH}

The propagation of a particle in the nuclear medium is often characterized by its mean free path $\lambda$. The experimental value obtained for medium energy nucleons $(100-600 \mathrm{MeV})$ is of order $5 \mathrm{fm}$ [20]. It has been shown, however, that this experimental value is ambiguous and depends critically on the parametrization chosen for the radial shape of the optical potential [21]. Simple microscopic models suggest a smaller value for $\lambda$, of order 1.5-3 $\mathrm{fm}$, in this energy range [20]. Negele and Yazaki [22] have included the effects of nonlocality in the evaluation of the self-energy for a nucleon propagating in nuclear matter. 
It was shown that these effects lead to an increase in the value of $\lambda$. Very recently Pandharipande and Pieper [23] have also discussed the Pauli blocking and nonlocality corrections to the evaluation of the nucleon mean free path in nuclear matter.

We deduce the nucleon mean free path from the derived equivalent local potentials according to

$$
\lambda(r)=-\frac{\sqrt{\hbar^{2}[E-V(r)] / 2 m}}{W(r)},
$$

where $V(r)$ and $W(r)$ are the real and the imaginary parts of the central potentials, $m$ is the nucleon mass and $E$ the incident energy.

In Fig. 9 we show the mean free path in the nuclear interior $\lambda(r=0)$ as a function of nucleon energy, calculated from the local equivalent potentials to the DSA (solid line) and SSA (dashed line) KMT potentials. As is clear from the figure, the second-order multiple scattering contributions increase considerably the value of the calculated mean free path. The values obtained, $\lambda=3-$ $4 \mathrm{fm}$ for 100-200 MeV incident energies, are compatible with those obtained by Pandharipande and Pieper [23] following the inclusion of both Pauli blocking and effective mass corrections in their nuclear matter calculations.

\section{CONCLUSIONS}

We have evaluated local phase equivalent potentials to calculations of the multiple scattering expansion of the $p$ ${ }^{16} \mathrm{O}$ optical potential at 100,135 , and $200 \mathrm{MeV}$ incident energies. The second-order KMT potential terms are found to reduce significantly the real and imaginary parts of the nucleon optical potential calculated from the firstorder term in the nuclear interior. These Pauli blocking corrections modify the shape of the potential in a nontrivial way. We have also evaluated an associated Perey factor for the multiple scattering optical potentials and find that the second-order calculations reduce the wave function in the nuclear interior by a factor of order 0.86 at $100 \mathrm{MeV}$. The departure of this factor from unity is

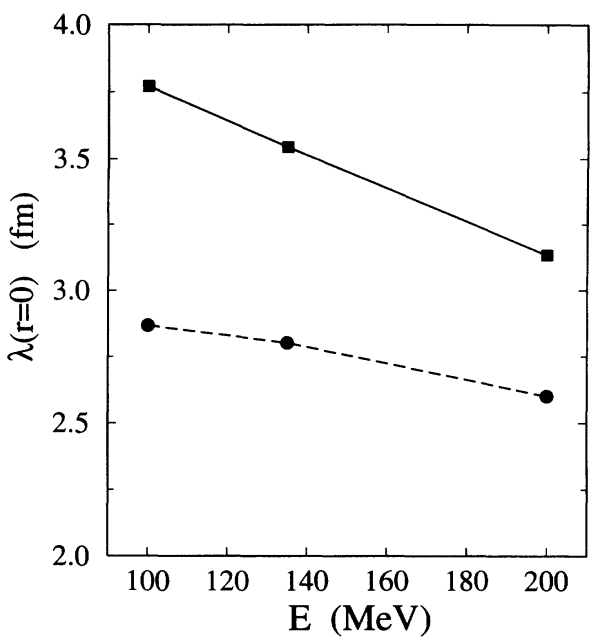

FIG. 9. Mean free path $\lambda(r=0)$ as a function of the incident nucleon energy calculated from the second-order (solid line) and first-order (dashed line) local equivalent potentials as described in the text.

reduced as the nucleon energy increases. The inclusion of second-order multiple scattering contributions to the optical potential are shown to predict a significant change in the calculated nucleon mean free path and we obtain $\lambda=3-4 \mathrm{fm}$ for an ${ }^{16} \mathrm{O}$ target at $100-200 \mathrm{MeV}$ incident energy.

\section{ACKNOWLEDGMENTS}

The financial support of the Instituto Nacional de Investigação Científica (INIC) and of the British Council which made this collaboration possible are gratefully acknowledged. The support of Junta Nacional de Investigação Científica e Técnológica (JNICT) through Grant 1094/CEN (for R.C.) and the Science and Engineering Research Council (UK) through Grants GR/G/53556, GR/H/53648 (for J.A.T. and R.C.J.) and through Grant $\mathrm{GR} / \mathrm{F} / 12501$ (for SGC) are also acknowledged.
[1] A.K. Kerman, H. McManus, and R.M. Thaler, Ann. Phys. 8, 551 (1959).

[2] H.F. Arellano, F.A. Brieva, and W.G. Love, Phys. Rev. C 41, 2188 (1990); H.F. Arellano, W.G. Love, and F.A. Brieva, Phys. Rev. C 43, 2734 (1991).

[3] Ch. Elster, T. Cheon, E.F. Redish, and P.C. Tandy, Phys. Rev. C 41, 814 (1990).

[4] C.R. Chinn, Ch. Elster, and R.M. Thaler, Phys. Rev. C 44, 1569 (1991).

[5] R. Crespo, R.C. Johnson, and J.A. Tostevin, Phys. Rev. C 41, 2257 (1990).

[6] L. Ray, G.W. Hoffmann, and W.R. Coker, Phys. Rep. 212, 223 (1992).

[7] R. Crespo, R.C. Johnson, and J.A. Tostevin, Phys. Rev. C 44, R1735 (1991); ibid. 46, 279 (1992); R. Crespo,
Ph.D. thesis, University of Surrey, 1991 (unpublished).

[8] R. Crespo, R.C. Johnson, and J.A. Tostevin, Phys. Rev. C 48, 351 (1993).

[9] E.D. Cooper, Nucl. Phys. A495, 483 (1989).

[10] R.A. Eisenstein and F. Tabakin, Comp. Phys. Commun. 12, 2367 (1976).

[11] R.H. Landau, Comp. Phys. Commun. 28, 1073 (1982).

[12] R.S. Mackintosh and A.M. Kobos, Phys. Lett. 116B, 95 (1982); A.A. Ioannides and R.S. Mackintosh, Nucl. Phys. A467, 482 (1987).

[13] S.G. Cooper and R.S. Mackintosh, Inverse Probl. 5, 707 (1989).

[14] S.G. Cooper and R.S. Mackintosh, Program IMAGo, Open University Report No. OUPD 9201, 1993 (unpublished). 
[15] J.P. Jeukenne, A. Lejeunne, and C. Mahaux, Phys. Rev. C 16, 80 (1977).

[16] F.G. Perey in Direct Interactions and Nuclear Reaction Mechanisms, edited by E. Clemental and C. Villi (Gordon and Breach, New York, 1963).

[17] F.G. Perey and B. Buck, Nucl. Phys. 32, 353 (1962).

[18] S.G. Cooper and R.S. Mackintosh, Nucl. Phys. A511, 29 (1990).

[19] H. Fiedeldey, R. Lipperheide, G. H. Rawitscher, and S.A
Sofianos, Phys. Rev. C 45, 2885 (1992).

[20] A. Nadasen et al., Phys. Rev. C 23, 1023 (1981).

[21] H.O. Meyer and P. Schwandt, Phys. Lett. 107B, 353 (1981).

[22] J.W. Negele and K. Yazaki, Phys. Rev. Lett. 47, 71 (1981).

[23] V.R. Pandharipande and S. C. Pieper, Phys. Rev. C 45, 791 (1992). 


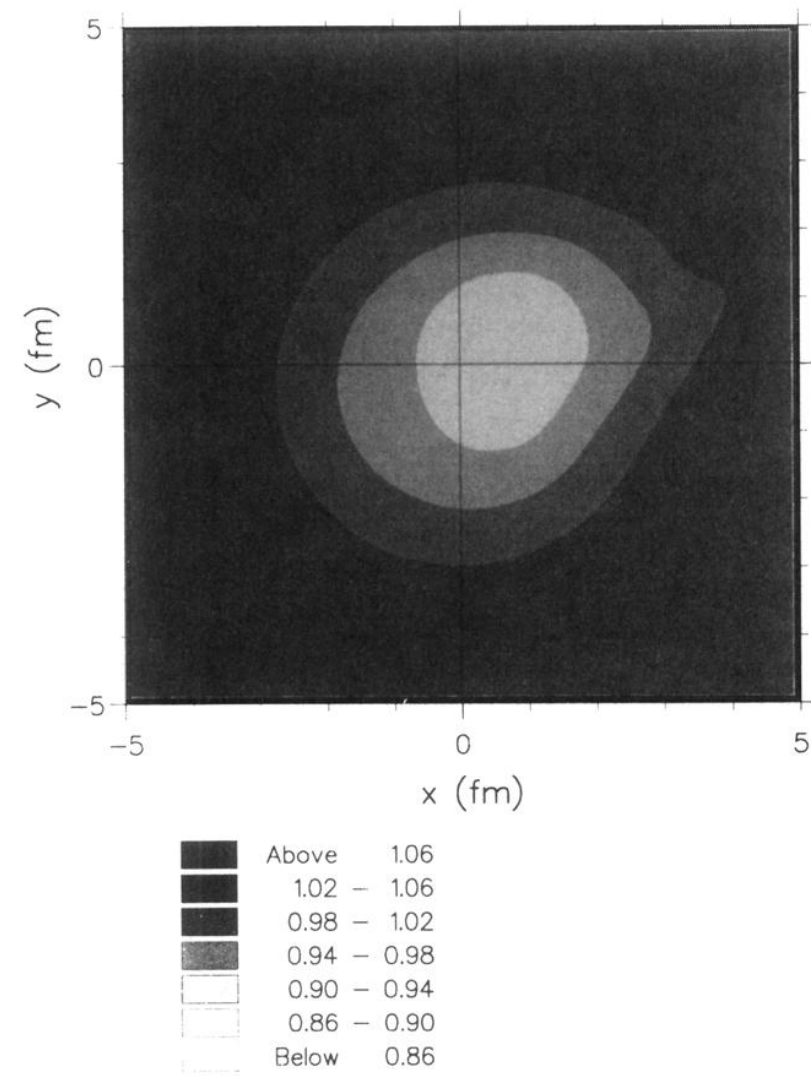

FIG. 4. Perey factor calculated from the first-order (SSA) optical potential at $100 \mathrm{MeV}$. 


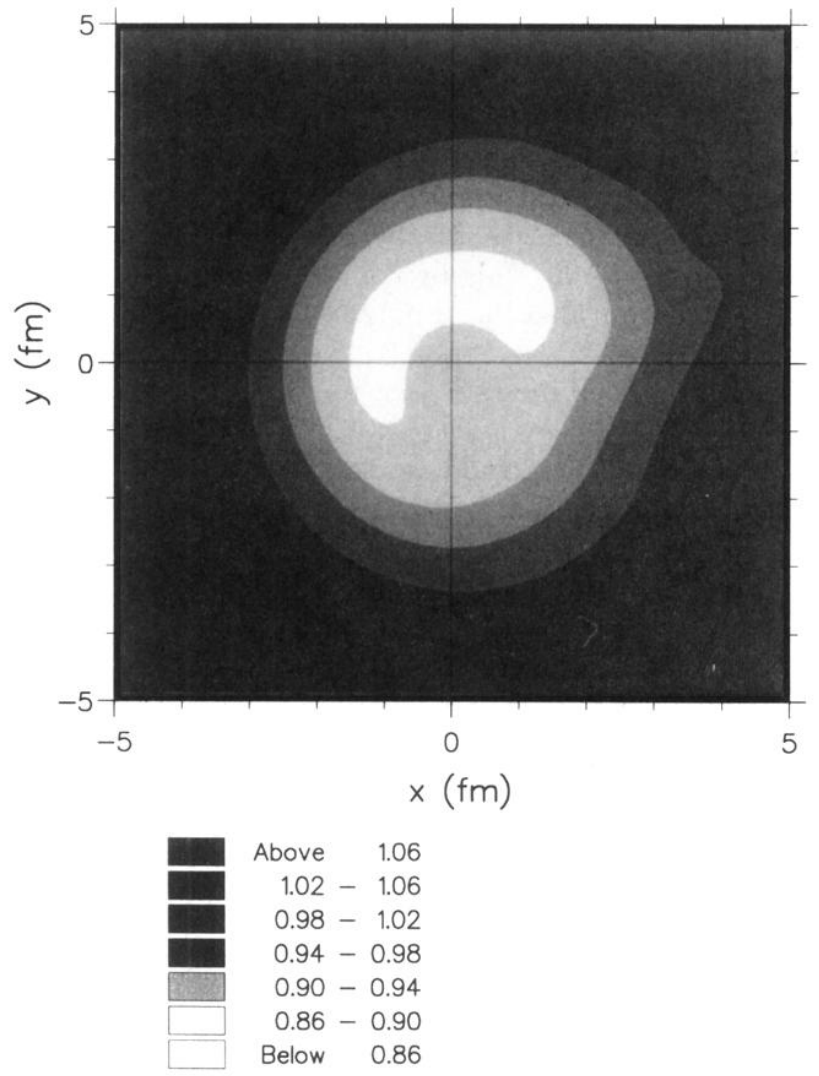

FIG. 5. Perey factor calculated from the second-order (DSA) optical potential at $100 \mathrm{MeV}$. 


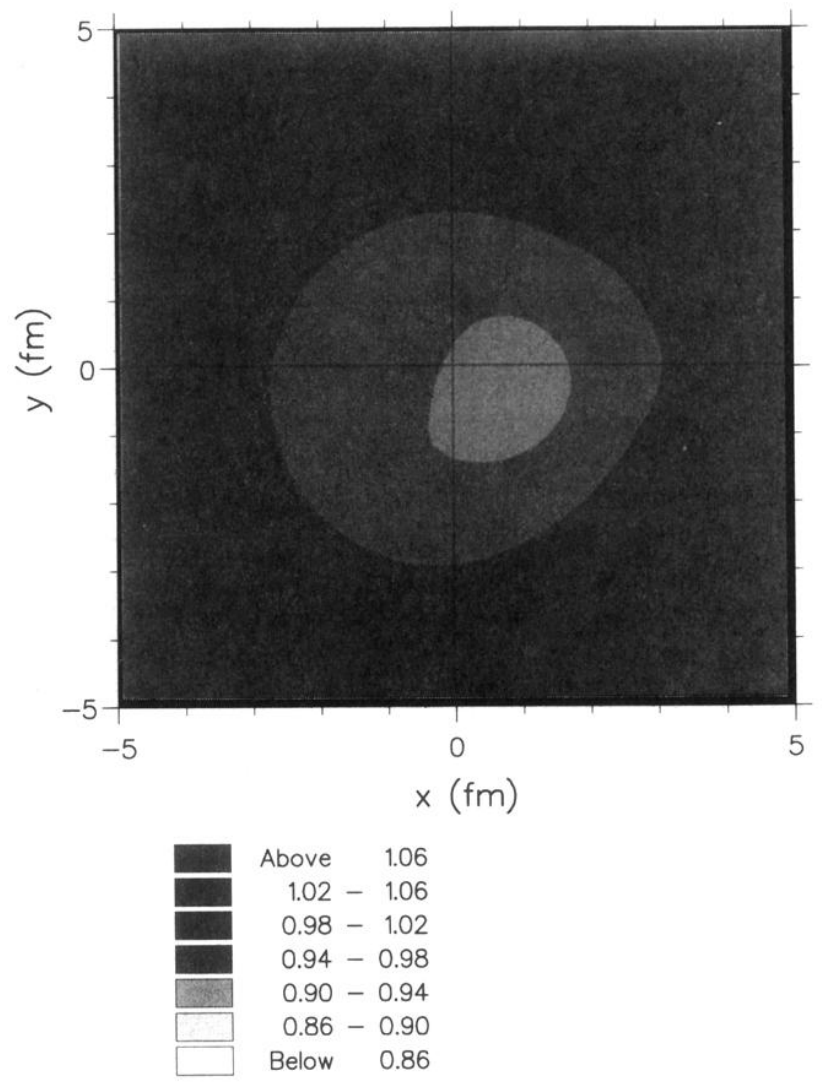

FIG. 6. Perey factor calculated from the first-order (SSA) optical potential at $200 \mathrm{MeV}$. 


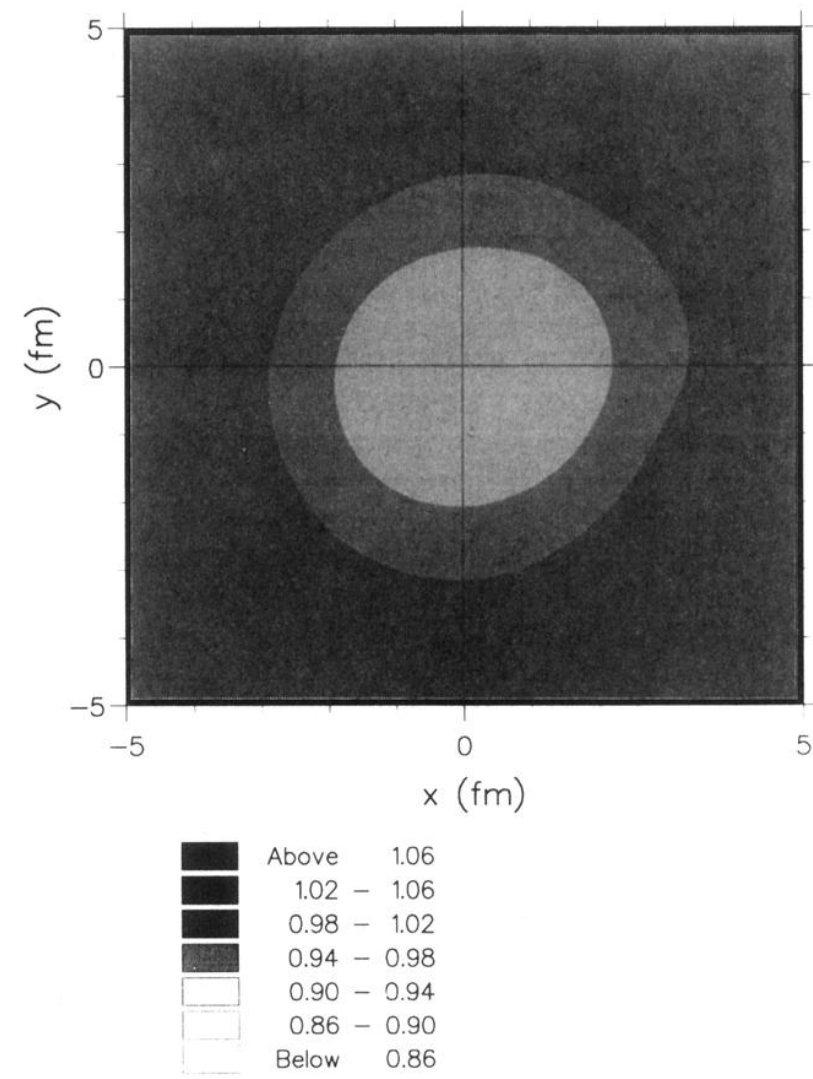

FIG. 7. Perey factor calculated from the second-order (DSA) optical potential at $200 \mathrm{MeV}$. 